\title{
Fast estimation of the phase diagram of a binary system using infrared thermography
}

\author{
by R. Cadoret*, E. Palomo del Barrio* and J. Daranlot** \\ *Univ. Bordeaux, I2M, UMR 5295, F-33400 Talence, France, elena.palomo@trefle.u-bordeaux.fr \\ ${ }^{* *}$ Solvay, 178 avenue du Dr Schweitzer, F-33608 Pessac, France
}

\begin{abstract}
Arial, 9pt, bold)
A method based on infrared thermography for fast estimation of the phase diagram of solid-liquid binary systems has been developed. The method involves heating simultaneously a set of small crystals of a system $A / B$, with compositions ranging from $0 \% \mathrm{~B} /(\mathrm{A}+\mathrm{B})$ to $100 \% \mathrm{~B} /(\mathrm{A}+\mathrm{B})$, and to observe their thermal behavior with an infrared camera. Recorded data are then analyzed by Singular Value Decomposition techniques to identify liquidus and solidus lines. Different binary systems has been investigated and the results achieved have been compared with the phase diagrams determined by standard DSC testing, as well as with the phase diagrams predicted by thermodynamic modeling.
\end{abstract}

\section{Introduction}

Solid-liquid phase change materials (PCM) are commonly used today for thermal energy storage applications. Most of the PCM in the market are either pure substances or multi-component systems, often binary systems, whose composition is chosen so that the melting point or the temperature range of melting is appropriate for the intended applications. The development of new PCM frequently involves the investigation of PCM's blends, because the mixtures would improve the performances (melting point, energy density, stability, etc.) of the components. First step in the study of a new mixture is the determination of the phase diagram of the mixture, which is used to identify the compositions of interest (if any).

Differential Scanning Calorimeter (DSC) is one of the most widely used experimental tools for phase change materials analysis. It allows determining transition temperatures as well as enthalpy changes. DSC can also be used to determine the phase diagram of a mixture, but the testing time will be rather long. Testing one-single blend at one-single composition requires three successive cycles of melting/crystallization at different heating rates $\left(0.1^{\circ} \mathrm{C} / \mathrm{min}, 0.5^{\circ} \mathrm{C} / \mathrm{min}\right.$ and $1^{\circ} \mathrm{C} / \mathrm{min}$ ) for accurate estimation of transition temperatures [1]. Assuming that the blend is tested at 20 different compositions, the time necessary for determining the phase diagram is approximately 10 days. Therefore, the DSC is not the ideal tool for investigating a large number of mixtures in a reasonable time, as it is often necessary in projects aimed at developing new PCM.

To reduce significantly the testing time and to accelerate the screening step when investigating a large number of mixtures, we have developed a new experimental device. It allows determining the phase diagram of one-single blend is less than 2 hours.

\section{The experimental method and the experimental set-up}

The proposed method aims at providing an approximate, but accurate enough estimation of the phase diagram of binary systems. The method involves heating simultaneously a set of small samples of a system $A / B$, with compositions ranging from $0 \% \mathrm{~B} /(\mathrm{A}+\mathrm{B})$ to $100 \% \mathrm{~B} /(\mathrm{A}+\mathrm{B})$, and to observe their thermal/optical evolution with an infrared camera. Recorded data are then analyzed to identify points of equilibrium between solid and liquid phases of each sample.

Accordingly, main elements of the experimental set-up are the infrared camera, the "sample-set" and the heating system. The "sample-set" consist of a series of small crystals ( $2-4 \mathrm{~mm}$ in diameter) of the binary system to be tested placed in a more or less regular manner on the surface of a thin plate. The plate is made of aluminum with highly polished faces so as to have low surface's emissivity in the infrared. The composition of the samples ranges from $0 \% \mathrm{~B} /(\mathrm{A}+\mathrm{B})$ to $100 \% \mathrm{~B} /(\mathrm{A}+\mathrm{B})$, with a pitch of $2-5 \%$ most times.

The "sample-set" is put on the heating system of the experimental set-up, and it is submitted to a constant heating rate up to $T_{\max }$. The temperature $T_{\max }$ is chosen so that all the samples in the "sample-set" melt during the experiment. The "sample-set" is imaged by the infrared camera all over the experiment with a frequency of $25 \mathrm{~Hz}$. One image typically includes $250 \times 210$ pixels. Simultaneously, the temperature of the samples' plate is measured with a thermocouple. Afterward, raw data are processed. Processing applied involves three main steps: 1) segmentation of the infrared images to isolate the regions of the plate occupied by the samples; 2) selection of the pixels of interest for each sample; and c) identification of the temperatures at which the sample's melting begins and ends. 


\section{Resultats}

Different binary systems has been used for testing and validation purposes. Results achieved have been compared with the phase diagrams determined by standard DSC testing, as well as with the phase diagrams predicted by thermodynamic modeling. The figure below provides an example. As it can be seen, the proposed method is capable of estimating the phase diagram of a binary system with sufficient accuracy for screening.

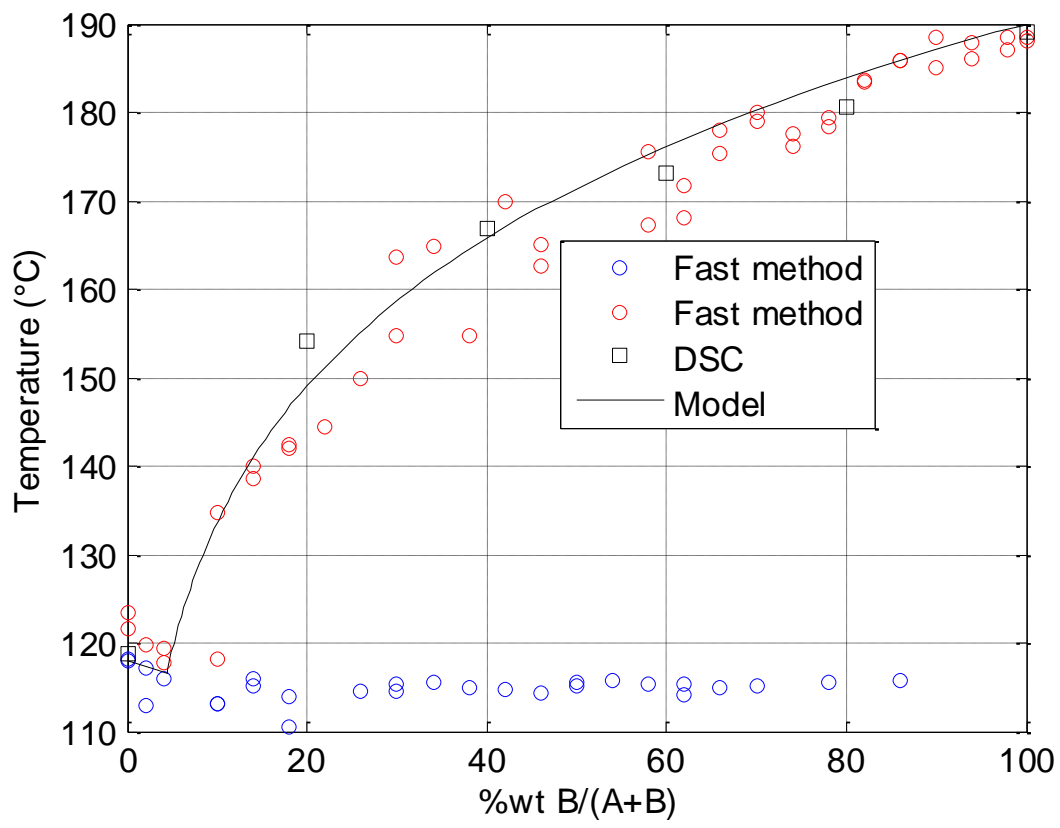

Phase diagram of a binary system determined by a) the experimental method proposed in this paper (red and blue symbols), b) DSC testing (black squares) and c) modeling (continuous line).

\section{Acknowledgment}

The authors acknowledge the financial support of the European Commission for subsidizing SAM.SSA (Sugar Alcohols based Materials for Seasonal Storage Applications) Project within the 7th framework program for research.

\section{REFERENCES}

[1] Kousksou T, Jamil A, Zeraouli Y, Dumas JP. Equilibrium liquidus temperatures of binary mixtures from differential scanning calorimetry. Chem. Eng. Sci. 2007, 62: 6516-6523. 